\title{
Dust in Warm Debris Disks
}

\author{
Hideaki Fujiwara* \\ Subaru Telescope, National Astronomical Observatory of Japan \\ E-mail: hideakienaoj.org
}

Debris disks were discovered in main-sequence stars by infrared excess over the photospheric emission in observations by IRAS in the 1980s. Since debris disks are thought to be formed as a consequence of the collision of planetesimals, or the destruction of cometary objects, it is interesting to examine mineralogical characteristics of debris dust and to explore connection between the debris dust and the dust in the solar system. Recent high-sensitivity observations in the mid-infrared allow us to investigate the properties of warm dust grains in the inner region of debris disks, which should have a more direct link to the formation of terrestrial planets than the low-temperature dust that has been previously studied. Here we introduce results of our survey of warm debris disks around main-sequence stars based on the AKARI All-Sky Survey. We also show mineralogical characteristics and far-infrared measurements of the extremely bright warm debris disk around HD 15407A, and discuss its origin.

The Life Cycle of Dust in the Universe: Observations, Theory, and Laboratory Experiments - LCDU 2013, 18-22 November 2013

Taipei, Taiwan

${ }^{*}$ Speaker. 


\section{Introduction}

IRAS observations discovered circumstellar dust disks (debris disks) around main-sequence stars. The disks were identified from their infrared (IR) excess (Figure W). The lifetime of primordial grains from the protoplanetary disk are much shorter than the age of the star around which these disks are observed, therefore the circumstellar dust grains around main-sequence stars are not primordial but supplied during planet formation process. Destruction of planetesimals or comets is a likely supply source of dust [W, [1]. In other words, debris dust is supplied from extrasolar analogues to the "asteroid belt" or the "Kuiper Belt" in the framework of our solar system. The orbital evolution of asteroids and Kuiper Belt Objects is strongly affected by giant planets like Jupiter. The production rate of debris dust by the destruction of small bodies in the "asteroid belt" or the "Kuiper Belt" and the orbital evolution of debris dust is also affected by the presence of planets [3]. Therefore, it is very important to study the relationship between the presence of planets and debris dust for the investigation of the mechanism of dust supply/dissipation around stars.

Most of the known debris disks only show excesses at wavelengths longer than $25 \mu \mathrm{m}$. These excesses comes from the thermal emission of dust grains with low temperatures $(\leq 100 \mathrm{~K})$ that exist at distance of several 10s of AU from the central star. To date, warm $(>200 \mathrm{~K})$ debris disks are rare and little is known about the properties of the warm debris disk material located close to the star, which has a more direct link to the formation of terrestrial planets than the low temperature debris [䧃]. Recent high-sensitivity surveys at 10-20 $\mu \mathrm{m}$ spotlight the importance of this inner debris disk material.

\section{AKARI Mid-Infrared Survey of Warm Debris Disks}

AKARI is a Japanese IR satellite dedicated primarily to an IR all-sky survey [G]. The MidInfrared (MIR) All-Sky Survey was performed using 9 and $18 \mu \mathrm{m}$ broad band filters with the IRC onboard AKARI until August 2008 [目], and a complementary FIR survey at 65-160 $\mu \mathrm{m}$ was performed with the FIS [ $\square]$. The data from the AKARI/IRC All-Sky Survey is very powerful at identifying warm debris disks and contributing to the number of sample with its higher sensitivity and spatial resolution than those of IRAS. [8] conducted a search for warm debris disks by taking cross-correlation between the AKARI MIR All-Sky Survey data, the 2MASS All-Sky Catalogue of Point Sources [Q] , and dwarf stars from the Tycho-2 Spectral Type Catalogue [ए]] and by extracting stars with larger $18 \mu \mathrm{m}$ excess based on observed $K_{\mathrm{S}}-[18]$ color.

24 candidates of warm debris disks have been identified from about 900 main-sequence stars in the data of the AKARI MIR All-Sky Survey (Figure [). Eight candidates were newly reported to show $18 \mu \mathrm{m}$ excess with the AKARI observations. Other two candidates were originally suggested to show infrared excess by IRAS observations [ए]] and confirmed by the AKARI study. Although those candidates were selected based on the $18 \mu \mathrm{m}$ excess, 13 stars also show excess at $9 \mu \mathrm{m}$, suggesting the presence of warmer dust around the stars.

From the IR SED of the warm debris disk candidates, dust temperature of the inner rim of the disk was derived assuming blackbody emission for the IR excess. The temperature was converted to an orbital radius of the disk based on the stellar luminosity. Figure $\square$ plots the distribution of dust temperature and radius by sorting the spectral type of the central stars from early to late type. It is 

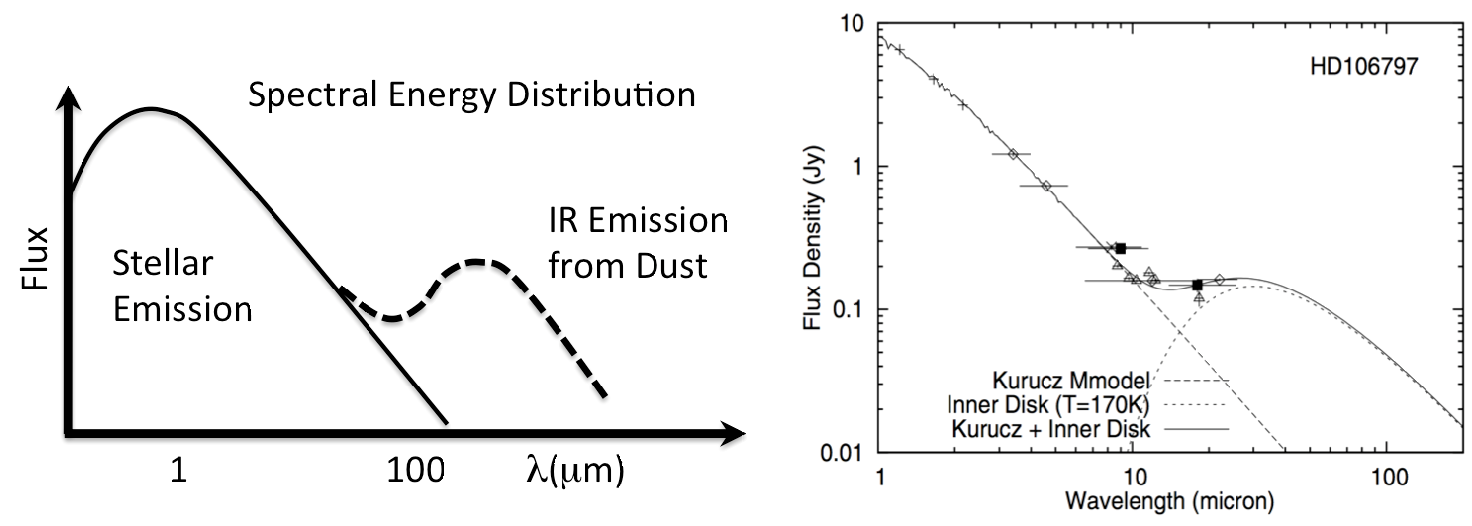

Figure 1: Left: Sketch of the SED of the debris disk. IR excess emission is seen over the photospheric level towards the debris disk. Right: An example of the SED of warm debris disk candidates discovered by AKARI (HD 106797), which was reported by [0, [2]].

seen that the investigated debris disks change their characteristics appreciably around the spectral type F0. The temperature is typically less than $200 \mathrm{~K}$ for A stars, while the temperature $>300 \mathrm{~K}$ for FGK stars. The radius of the inner rim of the disk is $>$ a few AU for A stars, while it is $<$ a few AU for FGK stars. The results suggest that warm dust is more abundant around FGK stars than around A stars. This difference can be explained by the effect of the radiation pressure on grains. For $\mu \mathrm{m}$-sized silicate dust around F0V stars, the radiation-pressure-to-gravity ratio $\beta=F_{\text {rad }} / F_{\text {grav }}$ gets close to unity. By taking the $\beta$ value and the absence of small dust grains around early-type stars into account, it is suggested that $\mu \mathrm{m}$-sized grains are blown out by the radiation pressure from A stars and that the difference in the disk characteristics between A and FGK stars seen in our sample may be driven by the radiation pressure on grains.

\section{Extreme Warm Debris Disk around HD 15407A}

Some warm debris disks identified by AKARI possess a large amount of dust and cannot be explained by steady-state evolution through planetesimal collisions. HD 15407A is an F3V main-sequence star (at $55 \mathrm{pc}$ ) with a warm debris disk having a large MIR excess selected from a search for debris disks using AKARI/IRC MIR All-Sky Survey data. This debris disk is extremely bright in the MIR and its fractional luminosity $\left(L_{\text {dust }} / L_{\text {star }}\right)$ is $\sim 0.005$ (comparable to $\beta$ Pic, the most famous debris disk), which cannot be accounted for by steady-state evolution of the disk by planetesimal collisions [[13] and thus requires transient events [[4]]. The dust temperature is estimated as 500-700 K, corresponding to a distance of 0.5-1.0 AU from the central star. The debris disk of HD 15407A may belong to a new sub-group of debris disks, in which violent dust supply and processing are taking place in the inner region $(<$ a few $\mathrm{AU})$ of the disk.

Significant features from silica $\left(\mathrm{SiO}_{2}\right)$ dust are seen in the spectrum obtained by Spitzer/IRS [144, [5]] (Figure [3). The presence of abundant silica dust is also detected around the A0V star HD 172555 by Spitzer/IRS spectroscopy [16]. As a probable source of silica dust around HD 172555, in which abundant $\mathrm{SiO}$ gas is also detected, giant hypervelocity impact between large rocky planetesimals is suggested by [16]. It may be similar to the one that formed the Moon (giant impact) or 

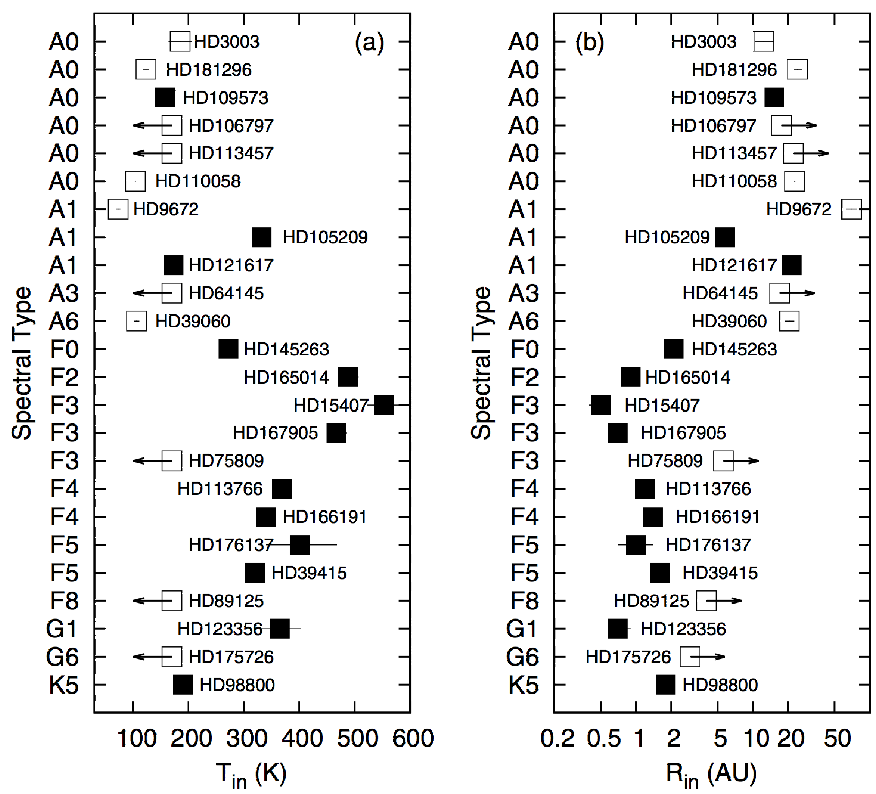

Figure 2: Distributions of dust temperature of the inner rim of the disk $\left(T_{\mathrm{in}}\right)$ (a) and orbital radius of disk $\left(R_{\mathrm{in}}\right)(\mathrm{b})$ of AKARI-identified warm debris disk candidates [ [ $]$ ]. Filled and open squares indicate the stars with and without $9 \mu \mathrm{m}$ excess, respectively. $T_{\text {in }}$ is set at $170 \mathrm{~K}$ for the stars without $9 \mu \mathrm{m}$ excess and photometric information at $\lambda \geq 24 \mu \mathrm{m}$.
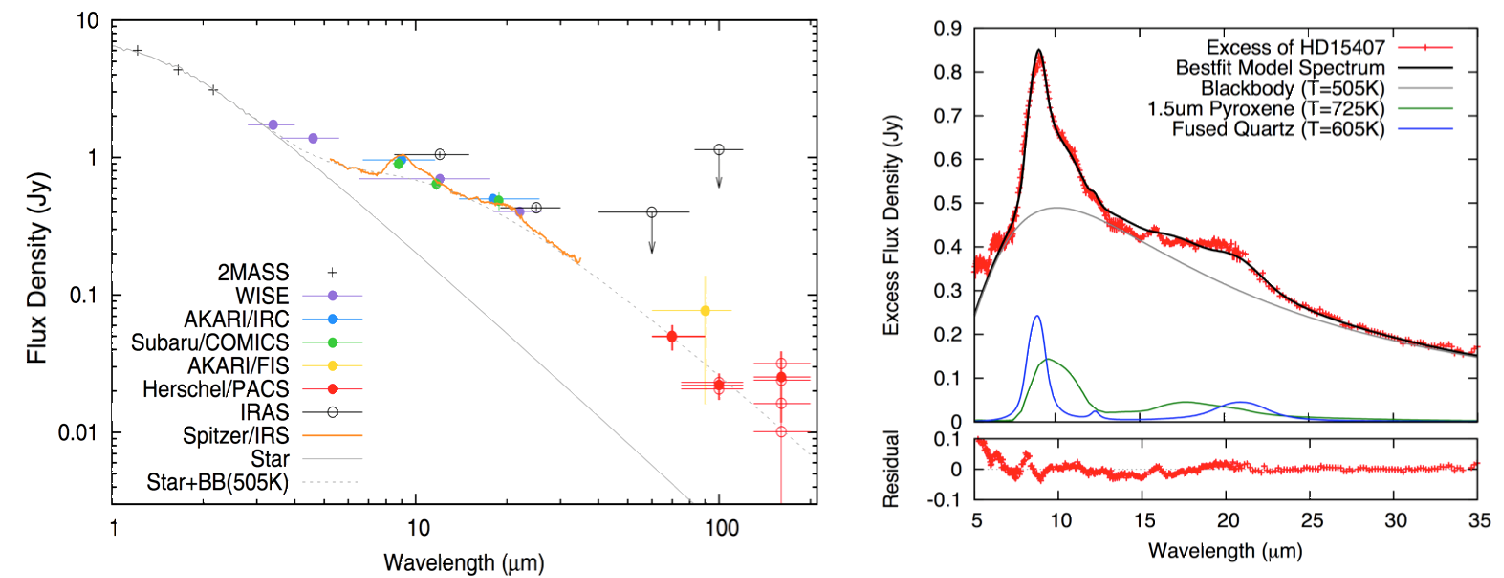

Figure 3: Left: IR SED of HD 15407A taken from [I]]. The photometric data of WISE, AKARI/IRC, Subaru/COMICS, and AKARI/FIS, and IRAS were shown together with the photospheric contribution of the star [ए8]. The orange solid and gray dashed lines indicate the Spitzer/IRS spectrum and the estimated continuum level of the excess emission, respectively, taken from [14]. Right: Debris disk spectrum of HD 15407 and the model spectrum that consists of blackbody dust, $1.5 \mu \mathrm{m}$-sized amorphous pyroxene, and fused quartz (silica). The most prominent component of the excess emission is blackbody, suggesting that the presence of a large amount of large-sized $(>5 \mu \mathrm{m})$ grains. The presence of silica is indicated by the broad features whose peaks are at $9.0 \mu \mathrm{m}$ and 20-21 $\mu \mathrm{m}$, while the presence of large amorphous pyroxene is suggested by the broad feature at $9-11 \mu \mathrm{m}$. The dust temperatures are estimated as $500-700 \mathrm{~K}$. 
the one that stripped the surface crustal material off of Mercury's surface. The comparable silicato-silicate ratio in $\mu \mathrm{m}$-sized small dust around HD 15407A suggests a similar origin of the silica dust with that of HD 172555. This also seems harmonic with the large fractional luminosity of HD 15407A's debris, which may be connected with a transient event.

[1]] reported follow-up FIR observations of HD 15407A at 60-160 $\mu \mathrm{m}$ by Herschel/PACS and AKARI/FIS. The observed flux densities of the debris disk in the FIR are clearly above the photospheric level of the star, suggesting excess emission at FIR as well as at MIR wavelengths. The observed FIR excess emission is consistent with the continuum level extrapolated from the MIR excess, suggesting that it originates in the inner warm debris dust and cold dust ( 50-130 K) is absent in the outer region of the disk. The absence of cold dust does not support a late-heavybombardment-like event as the origin of the large amount of warm debris dust around HD 15047A. Further theoretical studies in addition to observations with wide wavelength coverage will provide hints on the possible linkage between the large infrared excess, the dynamical evolution, and the possible transient event of the intriguing debris disk around HD 15407A.

\section{Summery}

We reviewed our recent research about dust in warm debris disks primarily based on AKARI and Spitzer observations. 24 candidates of warm debris disks have been identified in the data of the AKARI MIR All-Sky Survey. The investigated debris disks seem to change their characteristics appreciably around the spectral type F0, which may be driven by the radiation pressure on grains. Some warm debris disks identified by AKARI possess a large amount of dust and cannot be explained by steady-state evolution through planetesimal collisions. Particularly, the warm debris disk candidate around the F3V main-sequence star HD 15407A, whose fractional luminosity is $\sim 0.005$, shows the presence of abundant $\mu \mathrm{m}$-sized silica dust. It is suggested that the dust comes from violent collisional events. FIR observations of HD 15407A with AKARI and Herschel imply the absence of cold dust around HD 15407A, which does not support a late-heavy-bombardmentlike event as the origin of the large amount of warm debris dust around HD 15047A.

\section{References}

[1] D.E. Backman \& F. Paresce, Main-sequence stars with circumstellar solid material - The VEGA phenomenon in Protostars and Planets III, eds. E.H. Levy, \& J.I. Lunine, 1253 (1993).

[2] Kenyon \& Bromley, Terrestrial Planet Formation. I. The Transition from Oligarchic Growth to Chaotic Growth, AJ 1311837 (2005) [astro-ph/ 0503568 ].

[3] J.-C. Liou \& H.A. Zook, Signatures of the Giant Planets Imprinted on the Edgeworth-Kuiper Belt Dust Disk, AJ 118580 (1999).

[4] M.R. Meyer et al., Evolution of Mid-Infrared Excess around Sun-like Stars: Constraints on Models of Terrestrial Planet Formation, ApJ 673 L181 (2008) [arXiv: 0712 . 1057].

[5] H. Murakami et al., The Infrared Astronomical Mission AKARI, PASJ 59369 (2007)

[arXiv:0708.1796].

[6] D. Ishihara et al., The AKARI/IRC mid-infrared all-sky survey, A\&A 514 A1 (2010)

[arXiv:1003.0270]. 
[7] I. Yamamura et al., AKARI/FIS All-Sky Survey Point Source Catalogues.

[8] H. Fujiwara et al., AKARI/IRC $18 \mu \mathrm{m}$ survey of warm debris disks, A\&A $\mathbf{5 5 0}$ A45 (2013) [arXiv:1211.6365].

[9] R.M. Cutri et al., 2MASS All Sky Catalog of point sources.

[10] C.O. Wright et al., The Tycho-2 Spectral Type Catalog, AJ 125359 (2003).

[11] R.D. Oudmaijer et al., SAO stars with infrared excess in the IRAS Point Source Catalog, A\&AS 96625 (1992).

[12] H. Fujiwara et al., Hot Debris Dust Around HD 106797, ApJ 695 L88 (2009) [arXiv: 0903.1333$].$

[13] M.C. Wyatt et al., Transience of Hot Dust around Sun-like Stars, ApJ 698569 (2007) [astro-ph/0610102].

[14] H. Fujiwara et al., Silica-rich Bright Debris Disk around HD 15407A, ApJ 749 L29 (2012) [arXiv:1203.2534].

[15] J. Olofsson et al., Transient dust in warm debris disks. Detection of Fe-rich olivine grains, A\&A 542 90 (2012) [arXiv: 1204.2374$].$

[16] C.M. Lisse et al., Abundant Circumstellar Silica Dust and SiO Gas Created by a Giant Hypervelocity Collision in the 12 Myr HD172555 System, ApJ 7012019 (2009) [arXiv: 0906.2536 ].

[17] H. Fujiwara et al., The Absence of Cold Dust around Warm Debris Disk Star HD 15407A, ApJ 759 L18 (2012) [arXiv:1210.0587].

[18] R. L. Kurucz, Model Atmospheres for Population Synthesis, The Stellar Populations of Galaxies: Proceedings of the 149th Symposium of the International Astronomical Union, held in Angra dos Reis, Brazil, Kluwer Academic Publishers, Dordrecht (1992). 